\title{
Correction to: The Pulmonary Neuroepithelial Body Microenvironment
}

\section{Correction to:}

I. Brouns et al., The Pulmonary Neuroepithelial Body Microenvironment, Advances in Anatomy, Embryology and Cell Biology 233, https://doi.org/10.1007/978-3-030-65817-5

The original version of this book contained 5 individual chapters.

The content of the original 5 chapters has been merged into one single chapter: DOI https://doi.org/10.1007/978-3-030-65817-5_1.

In addition, the chapter title has been updated and abstract and keywords have been added to the chapter in this updated version. 